Workshop report

\title{
Best Practice Guidelines on molecular diagnostics in Duchenne/Becker muscular dystrophies
}

\author{
Stephen Abbs ${ }^{\mathrm{a}, *}$, Sylvie Tuffery-Giraud ${ }^{\mathrm{b}}$, Egbert Bakker ${ }^{\mathrm{c}}$, Alessandra Ferlini ${ }^{\mathrm{d}}$, \\ Thomas Sejersen ${ }^{\mathrm{e}}$, Clemens R. Mueller ${ }^{\mathrm{f}}$ \\ a DNA Laboratory, GSTS Pathology, Guy's Hospital, London SE1 9RT, UK \\ ${ }^{\mathrm{b}}$ Université Montpellier1, UFR Médecine and INSERM U827, Montpellier F-34000, France \\ ${ }^{\mathrm{c}}$ Department of Human and Clinical Genetics, LUMC, 2333 AL Leiden, The Netherlands \\ ${ }^{\mathrm{d}}$ Sezione di Genetica Medica, Dipartimento di Medicina Sperimentale e Diagnostica Università di Ferrara, Italy \\ ${ }^{\mathrm{e}}$ Karolinska Institute, Neuropediatric Unit, Stockholm 17176, Sweden \\ ${ }^{\mathrm{f}}$ Universität Würzburg, Institut für Humangenetik, Biozentrum, 97074 Wuerzburg, Germany
}

\section{Introduction}

A meeting of 29 senior scientists from Europe, the USA, India and Australia, was held in Naarden, The Netherlands on November 14-16, 2008, to establish consensus Best Practice Guidelines for molecular diagnosis of Duchenne and Becker muscular dystrophy (DMD/BMD). New therapeutic trials for DMD demand accurate diagnosis of the disorder, especially where the therapy is targeted towards specific mutations. These guidelines aim to help diagnostic laboratories attain that accuracy by describing the minimum standards for acceptable molecular diagnostic testing of DMD. For the different types of clinical referral received by a molecular diagnostic laboratory, the guidelines recommend the appropriate tests to be carried out, interpretation of the results and how those results should be reported.

\section{Internal and external quality control}

All laboratories offering molecular genetic testing for DMD/ BMD must follow established good laboratory practice, as documented for example in Guidelines for Quality Assurance in Molecular Genetic Testing, published by the Organisation for Economic Co-operation and Development [1].

In addition to following such guidelines, a laboratory should ideally demonstrate that it complies with internationally recognised standards for laboratory testing (e.g. ISO standards 15189: 2007 Medical laboratories - Particular requirements for quality and competence), by achieving formal accreditation with a member organisation of the International Laboratory Accreditation Cooperation (ILAC) or equivalent national accreditation body.

All tests should be validated/verified in individual laboratories prior to implementation; it is not acceptable to rely on the validation of a test by another laboratory, since that does not guarantee

\footnotetext{
* Corresponding author.

E-mail addresses: stephen.abbs@gsts.com (S. Abbs), sylvie.tuffery@inserm.fr (S. Tuffery-Giraud), e.bakker@lumc.nl (E. Bakker), fla@unife.it (A. Ferlini), thomas. sejersen@ki.se (T. Sejersen), crm@biozentrum.uni-wuerzburg.de (C.R. Mueller).
}

that it will perform accurately and reliably in all labs. A series of control samples representing all mutation types should therefore be collected by each laboratory to facilitate test validation/verification, and exchange of samples between laboratories is encouraged to allow this. External quality assessment schemes provide further validation of testing procedures and methods, and laboratories should participate in appropriate EQA schemes for DMD testing. If this is not possible, inter-laboratory exchange of samples should be arranged to compare and validate test results.

\section{Types of laboratory referral}

Patient samples are referred to clinical molecular genetic laboratories for the following investigations:

\subsection{Diagnosis in male patients}

Affected males suspected to have a dystrophinopathy based on high serum creatine kinase (CK) levels and/or muscle biopsy, are referred for a molecular confirmation of the clinical diagnosis (see Fig. 1). Molecular confirmation of a dystrophinopathy is achieved by demonstrating the presence of a clearly pathogenic variant in the $D M D$ gene. Absence of a $D M D$ mutation would reduce the likelihood of a patient having a dystrophinopathy, with the reduction being dependent on the sensitivity of the mutation screening procedure(s) used. It is currently not possible to refute a diagnosis of a dystrophinopathy based on the results of genetic testing, since no mutation detection protocol which is currently available can demonstrate $100 \%$ sensitivity.

From the patients' and relatives' perspectives, the speed with which a diagnosis can be made is extremely important in order to minimize anxiety and to reduce the risk of recurrence of the disease in the family. Therefore, in some instances a muscle biopsy and dystrophin analysis by immunohistochemistry might be needed to establish a quick and definite diagnosis.

Appropriate genetic counselling in the family is dependent on the knowledge of the diagnosis in the patient. This should be borne in mind when determining the appropriate procedures 


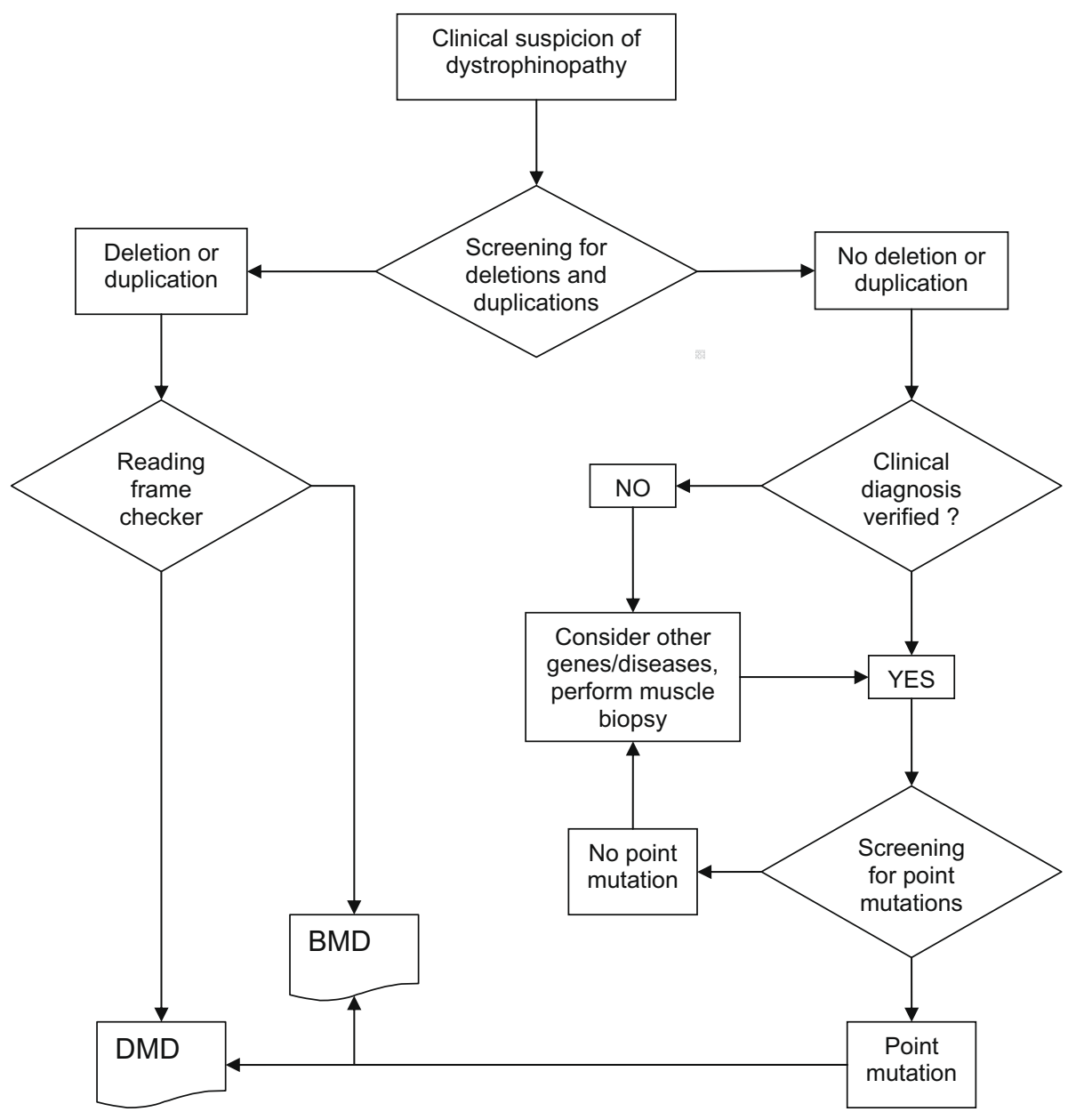

Fig. 1. Flow chart for the diagnostic work-up of a dystrophinopathy.

and methods to follow, and the approach may vary in different centres depending on the availability of different tests and facilities, and economic factors.

\subsubsection{Testing for deletions and duplications}

Since whole exon deletions are the predominant type of mutation in the DMD gene ( $65 \%$ ), an initial screen which detects the majority of deletions should be the minimum level of diagnostic test offered. A number of methods have been described, with the following being the most popular choices currently in use:

Multiplex PCR, to amplify the exons known to be most commonly deleted. The two PCR multiplex sets of Chamberlain et al. [2] and Beggs et al. [3], or recent improvements on these made by a number of centres, collectively enable the detection of about $98 \%$ of all DMD deletions. These two assays do not characterise the end points of all deletions, since they do not test all exons. Where the end point(s) of a deletion is (are) not determined by the multiplex PCR system being used, it is beneficial to use additional PCR assays to characterise the extent of the deletion, whenever possible. Details of PCR primers for all DMD exons can be found at www.dmd.nl

Quantitative assays of all exons offer an improvement in mutation detection rate, since they will detect all whole exon deletions, and additionally whole exon duplications ( $\sim 10 \%$ of DMD mutations). Further advantages are that these assays characterise the end points of most rearrangements (at the exon level of resolution), and can also be used for carrier testing of females. Of the quantitative methods available, multiplex ligation-dependent probe amplification (MLPA) [4] is currently the most widely used method among the workshop participants. Quantitative multiplex PCR of selected exons (e.g. [5]) and Southern blot hybridisation using cDNA probes [6] have both been widely used in the past, but have been superseded in many labs by the convenience and commercial availability of MLPA.

A recently developed quantitative approach to assay the $D M D$ gene with high resolution is array CGH (comparative genomic hybridisation) (e.g. [7-9]). This method uses thousands of oligonucleotides to interrogate copy number across the entire $2.2 \mathrm{MB}$ genomic region of the DMD gene including all exons and introns, and thereby maps rearrangement breakpoints to relatively narrow intervals depending on the spacing of the oligonucleotides at the breakpoints. It also can detect loss or gain of sequences at intronic breakpoints associated with some inversions and complex rearrangements, thereby offering a slightly higher mutation detection rate than MLPA and other exon-based tests.

If a method identifies an apparent single exon deletion or duplication based on the absence or increased amplification, respectively, of a single PCR amplification, or hybridisation, that result must be confirmed using an alternative assay. This different assay will verify whether the initial result could have been caused by a sequence variant (e.g. SNP), preventing hybridisation of a primer, probe, etc., or for duplications if the result was an anomaly. This can be achieved using the same method, for example with different primers to amplify an exon which looks deleted with multiplex PCR, or using a different method to assay that exon. High density array CGH has a further advantage here over most other methods 
which generate only a single result per exon, since most deletions or duplications are likely to be detected by several oligonucleotides on the array. This eliminates the possibility of a false positive result due to the presence of a SNP in a single probe or primer.

If a duplication of a single or multiple exons is identified it is important to test all exons for the possibility of additional exons being duplicated, since a number of apparently non-continuous duplications have been reported $[9,10]$.

When interpreting the deletion or duplication result (see below) if the predicted severity is discrepant with the observed clinical phenotype, it may be useful to repeat the tests on a second sample and/or to carry out additional laboratory tests using different methods, or offering more detail, in order to look for an explanation to the discrepancy. This additional work is not essential, and may not be possible in all centres.

\subsubsection{Testing for other mutation types}

If no deletion or duplication has been found, the clinical diagnosis cannot be confirmed nor excluded. If the clinical features, family history, and/or results of muscle biopsy suggest a dystrophinopathy, further tests should be offered to search for a pathogenic mutation. It is not expected that full mutation screening is available in every laboratory, but laboratories in which it is not available should seek an arrangement with another lab to carry out these further tests.

Numerous methods have been applied to scan the DMD gene for nucleotide changes, including: SSCP [11], dHPLC [12], FM-CSCE [13], PTT [14], HRM [15]. These pre-screens aim to offer a lower cost alternative to sequencing all the $79 \mathrm{DMD}$ exons, however, since the cost of sequencing has reduced dramatically over recent years it may be more appropriate to sequence the full gene now [16].

Sequencing can be performed on RT-PCR derived cDNA from muscle RNA, or on genomic DNA. If a pathogenic variant is identified in cDNA, it should be followed by sequencing of the appropriate region(s) in genomic DNA to confirm the result, and characterise the variant at the nucleotide level to allow future DNA-based testing in relatives. Note that for some variants identified in cDNA it may not be straightforward or even possible to characterise the sequence change at the genomic level, e.g. deep intronic mutations.

Complex rearrangements, or variants located deep into the large introns of the gene will not be detected using standard methods of DNA-based mutation screening, and RNA-based methods offer a reasonable likelihood of being able to detect them. These mutations appear to be of low frequency (approximately $2 \%$ of DMD mutations).

Next generation sequencing approaches also offer improved likelihood of being able to detect the full spectrum of DMD mutations, since the whole gene can be sequenced, including the introns. However, additional RNA-based studies may be required to evaluate the pathogenic effects of some of the many intronic variants likely to be detected.

In rare instances, the occurrence of more than one DMD mutation in a family has been reported (e.g. [17]). So, in extended pedigrees with more than one affected male, it may be wise to test all patients.

\subsection{Carrier diagnosis for a known familial mutation}

Carrier diagnosis of female relatives at risk of being heterozygotes for a known mutation can be conducted by most of the methods that have been used to identify the mutation in the index case of the family. Whenever possible, a sample from the index case (or a known carrier) should be run as a control sample or (at least) a written report on the mutation of the index patient should be available in order to avoid data transmission problems. Clearly, the method chosen to test female relatives must be capable of detecting the mutation in the heterozygous state, i.e. masked by the presence of the corresponding normal allele. Deletions therefore require either a quantitative method (such as MLPA or array $\mathrm{CGH}$ ), or a qualitative method, such as pulsed field or field inversion gel electrophoresis [18].

If the mutation of the index case cannot be found in genomic DNA from his mother, the frequency of germinal mosaics still confers a significant recurrence risk for future children (see below).

\subsection{Carrier detection for an unknown mutation}

If an affected male is not available to be tested, female relatives at risk of being carriers should be offered mutation testing, using any of the methods discussed above which are able to detect heterozygous mutations. Testing should start with the woman who has the highest prior carrier risk, usually the mother of an index case. An initial screen for deletions and duplications is a sensible first test, as in testing affected males. All the precautions associated with testing a male for deletions and duplications are equally applicable to testing females, such as the need to verify copy number changes involving only a single exon. If no mutation is found and before proceeding to more complex tests, it is recommended to review the available clinical information. With a strong clinical diagnosis and/or X-linkage, sequencing or other point mutation screening methods would be the ideal next step, but these may not be available in all laboratories. If these are not available in a laboratory, it is recommended that samples are forwarded to another laboratory which is able to conduct these tests. Haplotype analysis is an alternative (see below) if key family members are available.

\subsection{Diagnosis of manifesting carriers}

A small proportion of female carriers of DMD mutations exhibit clinical symptoms, some of which can be as severe as found in male patients. Norman and Harper [19] concluded that $2.5 \%$ of heterozygotes have muscle symptoms but the incidence of cardiomyopathy in female carriers may be higher $[20,21]$. Up to two thirds of carriers have persistently elevated serum CK levels [22]. Confirmation of diagnosis for these manifesting females follows the same principles as for affected males, and mutation screening follows the same principles as testing for carrier status of an unknown mutation. Clinical manifestation in females is believed to result from non-random X-inactivation. However, a few manifesting females have been identified with two mutations (A.F. and C.R.M. personal communication).

Chromosomal aberrations (45, X0; X-autosome translocations) should also be considered in fully manifesting girls.

\subsection{Prenatal diagnosis}

Prenatal diagnosis for DMD/BMD should only be carried out for male pregnancies. At present, it is not possible to predict whether a female heterozygote for a $D M D$ mutation will manifest any signs of the disorder or not, and therefore it would be inappropriate to offer prenatal diagnosis for a female fetus.

The familial mutation will preferably be known in advance of testing a male pregnancy, and should be confirmed before the prenatal test, or can be confirmed in parallel with testing the fetus by using the proband's DNA as a control. The same technique can be used to test for the mutation as was used to identify or verify the mutation in the proband. A check for maternal cell contamination (MCC) of the fetal DNA must be carried out, since its presence at a significant level may affect the interpretation of the fetal result. This would be a particular problem for example when testing a male fetus for a deletion using a non-quantitative PCR-based assay, 
since the presence of any maternal DNA will mask the presence of a deletion in an affected male fetus, resulting in a false negative diagnosis. A check for MCC can be done with markers from the dystrophin gene region (see Appendix) or with any polymorphic marker set used routinely in the laboratory, e.g. for identity testing. Further details and recommendations for checking for maternal cell contamination in prenatal samples can be found in the CMGS Best Practice Guidelines [23].

\subsection{Preimplantation genetic diagnosis (PGD)}

PGD is a specialist test carried out in a limited number of centres. For PGD, the same analytical considerations apply as for prenatal testing, but the special requirements of a PGD setting need to be considered, as documented in the Best Practice Guidelines from the European Society of Human Reproduction and Embryology PGD Consortium [24]. These tests will not be discussed further in this document.

\subsection{Haplotype analysis}

Haplotype analysis to trace the inheritance of high and low risk $D M D$ haplotypes within a family is an option for carrier detection and prenatal diagnosis which may be available in some laboratories. This method may be useful only if the family structure is suitable and the polymorphic markers used are informative. The results should be interpreted with care, particularly if the diagnosis is in any doubt.

At least three informative markers should be used, which should include a minimum of one intragenic marker and extragenic markers flanking the dystrophin gene on either side.

\section{Result interpretation}

\subsection{Deletions and duplications}

Whole exon deletions or duplications in the vast majority of cases can be considered to be pathogenic. The severity of clinical symptoms associated with these mutations does vary according to which exons are affected and the number of exons affected, but the principle factor which determines the severity is the effect that the mutation has on the reading frame. Deletions which disrupt the translational reading frame generally cause a severe (Duchenne) phenotype, whereas those which leave the reading frame intact have a less severe affect and result in the milder Becker phenotype. This reading frame hypothesis is consistent in about $92 \%$ of cases [25]. It should be used as a statistical basis for disease course prediction, but care should be taken when used for individual disease course prediction. The reading frame prediction is generally applicable to duplications, but should be used more cautiously because most techniques do not allow the determination of whether a duplication is arranged in a head-to-tail orientation. Additional tests may be carried out to evaluate the orientation of duplicated material, but such tests are not required for routine diagnosis. It may be important to determine this level of detail associated with duplication mutations if exon skipping therapy is to be carried out.

A reading frame checker is available at www.dmd.nl

\subsection{Other mutations}

With present knowledge, mutations leading to the generation of a premature stop codon are considered incompatible with fulllength protein synthesis and causative of a dystrophinopathy. However, a number of exceptions are on record in the DMD gene of exon skipping associated with nonsense-mutations (e.g. [26]).
Likewise, at the extreme $3^{\prime}$ end of the gene, nonsense-mutations may lead to a milder phenotype than expected.

Mutations within splice consensus sequences will almost invariably lead to aberrant splicing and should be checked for their effect on exon skipping and maintenance of the reading frame in the mRNA. Mutations in the immediate vicinity of splice consensus sites should be investigated by splice prediction programmes for their effect on splicing probability. If possible, muscle mRNA analysis should be performed or recommended.

Mutations leading to amino acid substitutions should be checked for their effect on protein structure and function by suitable algorithms (e.g. SIFT, Polyphen). With a huge, still not fully characterised protein like dystrophin, the limited power of such predictions should be recognised.

All sequence variants should be checked against the SNP databases (dbSNP) and the DMD gene mutation databases (www.dmd.nl; www.umd.be/DMD/W_DMD/index.html). For further recommendations about interpretation and reporting of unclassified sequence variants, see the Best Practice Guidelines published by CMGS and VKGL [27].

If a $D M D$ mutation has not been identified in a patient suspected of having a dystrophinopathy, alternative diagnoses should perhaps be considered, depending on the available clinical evidence and test results. Possible alternatives, especially for patients with milder clinical severities (referred with BMD), but also for patients referred with DMD at an early age, might include a limb girdle muscular dystrophy, or Emery Dreifuss muscular dystrophy. If, however, the diagnosis of DMD/BMD has been established by immunohistochemistry and/or immunoblotting, a second blood sample should be requested and analysed in order to rule out a sample swap.

\subsection{Haplotype analysis}

Haplotype analysis is often applied for families in which the causative mutation has not been identified. Individual haplotypes should be constructed from the observed marker alleles, and displayed in a pedigree drawing. Carrier and prenatal risks should be calculated, based on the segregation of the high and low risk haplotypes, using Bayesian methods [28]. The genetic distances of intragenic and flanking markers can be found at the DMD website (www.dmd.nl). In cases where a recombination is observed the calculations become more complex and may need to consider the likely location of an unknown mutation, based on the site of the recombination and relative likelihood of an unknown mutation residing on each side of the recombination. However, considerable caution must be taken with using linkage data for clinical determination of risks; it assumes the locus responsible for the disorder in a family is known (i.e. the DMD gene). Therefore the clinical diagnosis must be clearly defined as a dystrophinopathy, ideally by muscle biopsy analysis, but care should be taken in relying on linkage data to ascertain risks, especially in the absence of knowing the pathogenic mutation in a family. At least in case of a recombination, the risk of the proband should be calculated by suitable software (e.g. LINKAGE) or Bayesian logic.

With an established clinical diagnosis but in the absence of an identified DMD mutation, serum CK values of women at carrier risk are useful complementary information, making the risk calculations even more demanding, however.

\section{Result reporting}

\subsection{General points}

When writing the report, it should be borne in mind that the report may not be confined to the addressee but could be copied and 
forwarded to the patients and other health care professionals. Therefore, it should contain all necessary information to be comprehensible on its own.

The reason for referral should be re-stated, which at least specifies the type of test that was requested, e.g. diagnostic, carrier, or prenatal test. Reference to the laboratory tests carried out must include brief mention of the method(s) used and details of what was tested, e.g. which exons were screened. If a commercial kit is used (e.g. MLPA), the kit reference number(s) and version(s) should be documented.

For the sake of subsequent analyses to be performed in the family and for entry into patient registries it is recommended to use standard nomenclature for the reporting of mutations. For whole exon deletions and duplications, it is preferable to state in words which exons are deleted/duplicated, e.g. "deletion of exons 3-7", rather than use HGVS nomenclature. The HGVS nomenclature (www.hgvs.org) does not add any further information for these mutations, unless the breakpoints of the mutation have been characterised or at least narrowed down to a relatively small interval, in which case it would be appropriate to quote the mutation using full HGVS nomenclature. The report must clearly convey whether the end points of a deletion or duplication have been determined, so for example, the statement that a patient "has a deletion of exons 3-7" conveys that the end points are defined and exons 2 and 8 were present. If in this example exons 2 and 8 were not tested, the report should state that the patient "has a deletion which includes at least exons 3-7" and an additional statement that "the end points of the deletion have not been determined". The predicted effect of deletions on the reading frame should also be stated, clearly stating that it is a prediction. This can also be stated for duplications, but with greater caution since an assumption is generally made about the location and orientation of the duplicated sequence, as discussed above. If the deletion or duplication has been characterised in CDNA, the effect on the RNA can of course be given as a test result rather than a prediction.

For point mutations, HGVS recommended mutation nomenclature must be used, stating the sequence change at the DNA level (assuming it has been characterised in DNA), and (as predicted) in the protein. Also for clarity it is useful to state in words what the change is, and its predicted effect.

In all mutation reports, it is essential to quote the accession number of the $D M D$ reference sequence which has been used in classifying the mutation (e.g. NM_004006.2).

\subsection{Specific points to include in reports}

\subsubsection{Diagnostic tests}

5.2.1.1. Presence of mutation. Include details of the mutation (as discussed in Section 5.1).

State that the presence of the mutation confirms, or is consistent with, the diagnosis. If the patient's clinical phenotype (of either DMD or BMD) is consistent with the predicted severity of the mutation, the report can state specifically that the result confirms, or is consistent with, either DMD or BMD, or a female carrier of DMD or BMD. If the genotype - phenotype is discordant, or if the severity of the clinical phenotype is not specified in the referral, the report can refer to a dystrophinopathy, rather than specifically DMD or BMD.

Depending on local practice, it may be appropriate to offer carrier and prenatal tests to appropriate female relatives, and/or suggest referral for genetic counselling. Also, it may be useful in a separate document to the diagnostic report to inform patients about the possibility of entering their mutation on a national DMD Registry (e.g. www.dmdregistry.org) if they wish to be considered for therapeutic trials at any point in the future.
5.2.1.2. Absence of mutation. The report should state that the result neither confirms nor excludes the diagnosis, but it may be appropriate to state that the diagnosis of DMD, BMD, or a dystrophinopathy, is less likely. The sensitivity of the tests must be given, with references if appropriate. Further tests, if available, should be offered such as sequencing, immunohistochemical analysis, or testing of other genes, or if not available referral to another centre should be offered. Haplotype analysis may be offered if required for carrier or prenatal testing, if the family history or muscle biopsy tests suggest a dystrophinopathy, and if the family structure is suitable.

If an observed sequence variant cannot be classified according to disease causality, the report should clearly state this and also indicate that this result does not provide a basis for carrier testing and prenatal diagnosis in the family.

\subsubsection{Carrier tests}

5.2.2.1. Presence of mutation. The report should state that the presence of a mutation confirms the individual is a carrier of DMD, BMD, or a dystrophinopathy (as appropriate, see discussion above). Again, according to local practice, prenatal diagnosis might be offered, and carrier testing and genetic counselling may be offered to other appropriate relatives.

5.2.2.2. Absence of mutation. When the familial mutation is not present in a carrier test the report should clearly state that the female tested is not a carrier of the familial mutation. The exception to this would be if the individual is the mother of an affected boy(s) with no previous family history of the disorder (sporadic cases). In this case, the report must discuss the possibility that the mother may be mosaic for the mutation, and offer prenatal diagnosis if appropriate. The recurrence risk for offspring from the possibility of being mosaic should be stated, quoting recent published data which estimates a risk of about $9 \%$ associated with the high risk haplotype [29].

If a female has no recurrence risk associated with the familial mutation, it may be appropriate, depending on the nature of the referral, to state that prenatal diagnosis or further carrier testing of daughters are not indicated.

When the familial mutation is not known, and a mutation screen has failed to identify a mutation in a potential carrier, the carrier risk will be reduced depending on the detection rate of the molecular tests performed. This reduction should be calculated using Bayesian methods, and quoted on the report.

\subsubsection{Prenatal tests}

5.2.3.1. Presence of mutation. The implications of the presence of a mutation in a prenatal diagnosis must be reported clearly, stating that the (male) pregnancy is predicted to be affected with DMD, BMD, or a dystrophinopathy (as appropriate). The report should state that a significant level of maternal cell contamination has been excluded. If the mother does not carry the mutation somatically, this result may show that she is a germline mosaic for the mutation. This should be documented in the report, additionally stating that the recurrence risk in future pregnancies is increased accordingly.

5.2.3.2. Absence of mutation. Absence of the familial mutation in a prenatal diagnosis, requires the report to state that the (male) pregnancy is predicted to be unaffected by DMD, BMD, or dystrophinopathy (as appropriate) caused by the familial mutation. If the mother is a full carrier of the mutation, this prenatal result will itself exclude maternal cell contamination of the fetal DNA, and no additional tests for MCC will be required. The report should still state that a significant level of MCC has been excluded. 


\section{Meeting participants}

Stephen Abbs, London, UK

Egbert Bakker, Leiden, The Netherlands

Ligia Barbarii, Bucharest, Romania

Therese Bradley, Glasgow, Scotland

Kate Bushby, Newcastle, UK (ENMC)

George Christopoulos, Nicosia, Cyprus

Rosário dos Santos, Porto, Portugal

Morten Duno, Copenhagen, Denmark

Nihan Erginel-Unaltuna, Istanbul, Turkey

Javier Garcia-Planells, Valencia, Spain

Ieke Ginjaar, Leiden, The Netherlands

Madhuri Hegde, Atlanta, USA

Elena Ionica, Bucharest, Romania

Bremadesam Lakshmi, Chennai, India

Tanja Lalic, Belgrade, Yugoslavia

Ilona Lind, Tartu, Estonia

Marija Meznaric-Petrusa, Ljubljana, Slovenia

Clemens Mueller, Wuerzburg, Germany

Marcella Neri, Ferrara, Italy

Torbjörn Olausson, Gothenburg, Sweden

Henriett Piko, Budapest, Hungary

Bernd Rautenstrauss, München, Germany

Edwin Reyniers, Antwerp, Belgium

Emma Ryan, Dublin, Ireland

Thomas Sejersen, Stockholm, Sweden

Peter Taylor, Sydney, Australia

María Jose Trujillo-Tiebas, Madrid, Spain

Sylvie Tuffery-Giraud, Montpellier, France

Nikolaos Vogiatzakis, Athens, Greece.

\section{Acknowledgements}

The workshop was jointly organised and sponsored by The European Molecular Genetics Quality Network (www.emqn.org); EuroGentest (www.eurogentest.org); EU Contract no. FP6-512148); TREAT-NMD (www.treat-nmd.org); EU Contract no. FP6-036825), and hosted by the European Neuro-Muscular Centre (www. enmc.org).

\section{Appendix A}

URLS of databases and resources:

dbSNP: http://www.ncbi.nlm.nih.gov/projects/SNP/

Human Genome Variation Society (for mutation nomenclature

recommendations): www.hgvs.org

Leiden DMD database: www.dmd.nl

French Universal Mutation Database DMD: www.umd.be/DMD/

W_DMD/index.html

Polyphen: http://genetics.bwh.harvard.edu/pph/

SIFT: http://sift.jcvi.org/

List of polymorphic markers: www.dmd.nl

\section{References}

[1] OECD. Guidelines for quality assurance in molecular genetic testing; 2007.

[2] Chamberlain JS, Gibbs RA, Ranier JE, Nguyen PN, Caskey CT. Deletion screening of the Duchenne muscular dystrophy locus via multiplex DNA amplification. Nucleic Acids Res 1988;16:11141-56.
[3] Beggs AH, Koenig M, Boyce FM, Kunkel LM. Detection of 98\% of DMD/ BMD gene deletions by polymerase chain reaction. Hum Genet 1990;86: $45-8$

[4] Schouten JP, McElgunn CJ, Waaijer R, Zwijnenburg D, Diepvens F, Pals G. Relative quantification of 40 nucleic acid sequences by multiplex ligationdependent probe amplification. Nucleic Acids Res 2002;30:e57.

[5] Yau SC, Bobrow M, Mathew CG, Abbs SJ. Accurate diagnosis of carriers of deletions and duplications in Duchenne/Becker muscular dystrophy by fluorescent dosage analysis. J Med Genet 1996;33:550-8.

[6] Darras BT, Harper JF, Francke U. Prenatal diagnosis and detection of carriers with DNA probes in Duchenne's muscular dystrophy. $N$ Engl J Med 1987;316:985-92.

[7] Hegde MR, Chin EL, Mulle JG, Okou DT, Warren ST, Zwick ME. Microarraybased mutation detection in the dystrophin gene. Hum Mutat 2008;29:1091-9.

[8] del Gaudio D, Yang Y, Boggs BA, et al. Molecular diagnosis of Duchenne/Becker muscular dystrophy: enhanced detection of dystrophin gene rearrangements by oligonucleotide array-comparative genomic hybridization. Hum Mutat 2008;29:1100-7.

[9] Bovolenta M, Neri M, Fini S, et al. A novel custom high density-comparative genomic hybridization array detects common rearrangements as well as deep intronic mutations in dystrophinopathies. BMC Genomics 2008;9:572.

[10] White SJ, Aartsma-Rus A, Flanigan KM, et al. Duplications in the DMD gene. Hum Mutat 2006;27:938-45.

[11] Mendell JR, Buzin CH, Feng J, et al. Diagnosis of Duchenne dystrophy by enhanced detection of small mutations. Neurology 2001:57:645-50.

[12] Bennett RR, den Dunnen J, O'Brien KF, Darras BT, Kunkel LM. Detection of mutations in the dystrophin gene via automated DHPLC screening and direct sequencing. BMC Genet 2001;2:17.

[13] Ashton EJ, Yau SC, Deans ZC, Abbs SJ. Simultaneous mutation scanning for gross deletions, duplications and point mutations in the DMD gene. Eur J Hum Genet 2008:53-61.

[14] Roest PAM, Roberts RG, Van Der Tuijn AC, Heikoop JC, Van Ommen GJB, Den Dunnen JT. Protein truncation test (PTT) to rapidly screen the DMD-gene for translation-terminating mutations. Neuromusc Disord 1993;3:391-4.

[15] Almomani R, van der Stoep N, Bakker E, den Dunnen JT, Breuning $M H$ Ginjaar IB. Rapid and cost effective detection of small mutations in the DMD gene by high resolution melting curve analysis. Neuromuscul Disord 2009:383-90.

[16] Flanigan KM, von Niederhausern A, Dunn DM, Alder J, Mendell JR, Weiss RB. Rapid direct sequence analysis of the dystrophin gene. Am J Hum Genet 2003;72:931-9.

[17] Mostacciuolo ML, Miorin M, Vitiello L, et al. Occurrence of two different intragenic deletions in two male relatives affected with Duchenne muscular dystrophy. Am J Med Genet 1994;50:84-6.

[18] den Dunnen JT, Bakker E, van Ommen GJ, Pearson PL. The DMD gene analysed by field inversion gel electrophoresis. Br Med Bull 1989;45:644-58.

[19] Norman A, Harper P. A survey of manifesting carriers of Duchenne and Becker muscular dystrophy in Wales. Clin Genet 1989;36:31-7.

[20] Politano L, Nigro V, Nigro G, et al. Development of cardiomyopathy in female carriers of Duchenne and Becker muscular dystrophies. JAMA 1996;275:1335-8.

[21] Hoogerwaard EM, van der Wouw PA, Wilde AA, et al. Cardiac involvement in carriers of Duchenne and Becker muscular dystrophy. Neuromuscul Disord 1999;9:347-51.

[22] Emery AE. Clinical and molecular studies in Duchenne muscular dystrophy. Prog Clin Biol Res 1989;306:15-28.

[23] Clinical Molecular Genetics Society. Practice guidelines for the testing for maternal cell contamination (MCC) in prenatal samples for molecular studies. E-publication; 2008.

[24] ESHRE PGD Consortium. Best practice guidelines for clinical preimplantation genetic diagnosis (PGD) and preimplantation genetic screening (PGS). Hum Reprod 2005;20:35-48.

[25] Koenig M, Beggs AH, Moyer M, et al. The molecular basis for Duchenne versus Becker muscular dystrophy: correlation of severity with type of deletion. Am J Hum Genet 1989;45:498-506.

[26] Ginjaar IB, Kneppers AL, v d Meulen JD, et al. Dystrophin nonsense mutation induces different levels of exon 29 skipping and leads to variable phenotypes within one BMD family. Eur J Hum Genet 2000;8:793-6.

[27] Clinical Molecular Genetics Society and Dutch Society of Clinical Genetic Laboratory Specialists. Practice guidelines for the interpretation and reporting of unclassified variants (UVs) in clinical molecular genetics. E-publication; 2008.

[28] Bridge P. The calculation of genetic risks, 2nd ed. Johns Hopkins Press; 1997.

[29] Helderman-van den Enden AT, de Jong R, den Dunnen JT, et al. Recurrence risk due to germ line mosaicism: Duchenne and Becker muscular dystrophy. Clin Genet 2009;75:465-72. 